\title{
The analysis of technical object functioning stability as per the criterion of monitored parameters multivarite dispersion
}

\author{
V.N. Klyachkin ${ }^{1}$, I.N. Karpunina ${ }^{2}$ \\ ${ }^{1}$ Ulyanovsk State Technical University, 432027, Ulyanovsk, Russia \\ ${ }^{2}$ Ulyanovsk Civil Aviation Institute, 432071, Ulyanovsk, Russia
}

\begin{abstract}
The assessment of any technical object functioning stability is often limited by the monitoring of midrange constancy and monitored parameters dispersion. For that, the methods of multivariate statistical monitoring, used for the assessment of process stability, are offered. Midrange multivariate process monitoring is accomplished with the help of algorithms, based on Hotelling's chart statistics. While assessing the dispersion stability, one can use generalized variance based algorithms - covariance matrix determinant. The approaches described here to increase the efficiency of multivariate dispersion monitoring.
\end{abstract}

Keywords: multivariate statistical monitoring; generalized variance; specialized structures; exponentially weighted moving average

\section{Introduction}

Technical object functioning stability often testifies to its serviceability. Destabilization may immediately lead to a failure or emergency situation [1]. The fault fastest detection is our main task. For example, the hydraulic unit vibration monitoring is done with the help of the chain of detectors [2]. The reading of these detectors indicates the stability or instability of the monitored hydraulic unit operation. In water purifying system potable water physical -chemical properties are monitored (color index, chlorides and aluminum content, etc) [3]: it is vitally important to keep the properties within the limits.

Destabilization appears as the alternation of statistical midrange characteristics and monitored parameters dispersion, so to detect the fault, process statistical monitoring methods and algorithms could be used [4-6]. The most frequent destabilization features, connected with midrange changes, are either step -wise displacement or trend i.e. gradual midrange decrease or increase. To detect this type of destabilization, monitoring a single parameter, Shewart's charts for midrange values and individual observation are used. To monitor multiple correlated parameters, algorithms based on Hotelling's chart statistics are used. To increase the efficiency of Hotelling's chart, there are several methods offered. [7].One of them is finding specialized structures in the chart, probability of which is commensurate with the probability of false warning: trends, dramatic changes, events of approaching the control lines or abscissa. One more approach is the use of alert control line: several points in a row between the control lines show the availability of a midrange destabilization.

Similar methods could be used to find destabilization in investigating multivariate dispersion of object function parameters. The main fault types detected in object operation as per dispersion criterion are step-wise or gradual increase in monitored parameters dispersion. Monitoring one parameter the dispersion is characterized by a swing, standard deviation or variance. The main feature of multivariate dispersion is generalized variance- covariance matrix determinant.[8,9]. Sometimes effective variance is used [10].

There is a method of object operation stability analysis as per multivariate dispersion criteria, including the analysis of the detectors reading under the conditions of steady ( flawless) object operation, covariance matrix assessment; the selection of possible statistical tools for the future dispersion monitoring; the assessment of average run length for various statistical tools, taking into account all possible deviations; statistical tests; minimum run length tools selection; constant monitoring of object operation with the goal of multivariate dispersion stability diagnostics. The up-dated information technologies and modern software products enable fast diagnostics of object operation fault with the help of the developed algorithms.

\section{Generalized variance based algorithm for monitoring multivariate dispersion}

To verify the hypothesis about the equality of covariance matrix $\boldsymbol{\Sigma}$ to selected value $\boldsymbol{\Sigma}_{0}$, generalized variance, i.e. covariance matrix determinant, could be used $[4,8]$. For each time moment $t$ selected covariance matrix $\mathbf{S}_{t}$ is generated, the elements of which are as follows:

$$
s_{j k t}=\frac{1}{n-1} \sum\left(x_{i j t}-\bar{x}_{j}\right)\left(x_{i k t}-\bar{x}_{k}\right)
$$

$x_{i j t}$ is the result of $i$-observation as per $j$-exponent in $t$-sample $(i=1, \ldots, n, n$-sample size, $j, k=1, \ldots, p, p$ - number of monitored parameters, $t=1, \ldots, m, m$ - number of samples, taken to analyze the process as per learning sample). The matrix determinant (1) $\left|\mathrm{S}_{t}\right|$ is generalized variance of $t$ instantaneous sample.

The assessment of averagecovariance is computed as per the collection of samples too.

$$
\bar{s}_{j k}=\frac{1}{m} \sum_{t=1}^{m} s_{j k t},
$$

which make covariance matrix $S$; its determinant $|S|$ is used as the assessment of destination generalized variance $\left|\Sigma_{0}\right|$. While plotting the control chart the selected values of the generalized variance $\left|\mathrm{S}_{t}\right|$ for each $t$-sample are singled out on it.

The control lines of the generalized variance chart are determined from the ratios: 
$\left.\begin{array}{l}U C L \\ L C L\end{array}\right\}=\left|\Sigma_{0}\right|\left(b_{1} \pm u_{1-\alpha / 2} \sqrt{b_{2}}\right)$,

where $u_{1-\alpha / 2}$ is normal inverted distribution of order $1-\alpha / 2, \alpha$ is a confidence level (probability of false alert); the coefficients are computed as per the following formulae :

$b_{1}=\frac{1}{(n-1)^{p}} \prod_{j=1}^{p}(n-j) ;$
$b_{2}=\frac{1}{(n-1)^{2 p}} \prod_{j=1}^{p}(n-j)\left[\prod_{k=1}^{p}(n-k+2)-\prod_{k=1}^{p}(n-k)\right]$,

the assessment of destination generalized variance $\left|\Sigma_{0}\right|$ is found as per the learning sample .If the lower control line $L C L$ as per formula (3) is negative, zero value is taken.

Destabilization of the process is witnessed by at least one point getting beyond one of the control lines on the chart of the generalized variance, i.e. the process is steady when the in equation below is satisfied:

$$
L C L<\left|\mathbf{S}_{t}\right|<U C L
$$

where $t$ is the number of monitored samples. For example, Fig.1 shows the chart of generalized variance: lower control line is zero, no points beyond the control line: the process is steady.

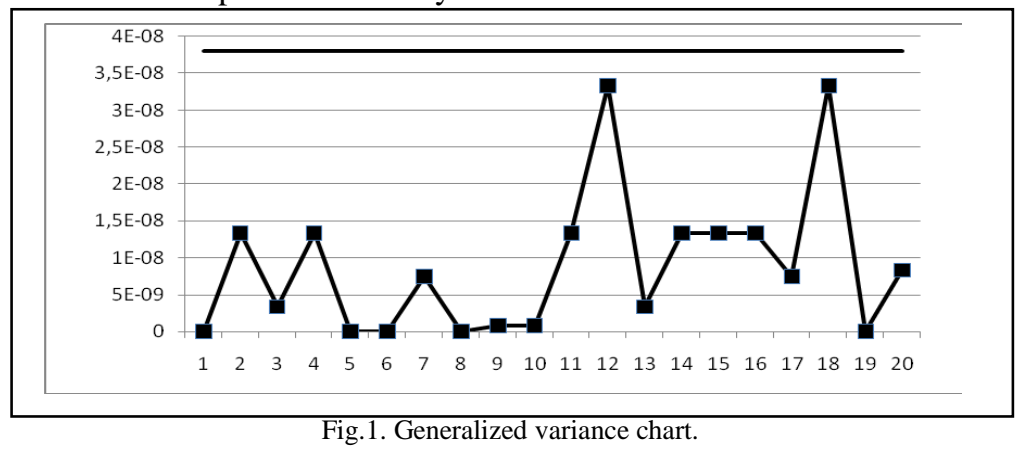

\section{Methods to improve efficiency of faults detection as per multivariate dispersion}

\subsection{Searching the structures of special form}

The process is considered steady as per criteria of multivariate dispersion if on the chart of generalized variance there are no points beyond the control lines, i.e. the condition is followed (6). This condition is important, but very often insufficient to ensure the process stability. Sometimes on the chart there are special form structures, which testify process instability: these are the structures, the probability of which is commensurate with the probability of false alert. For example, several successive points increasing or decreasing indicate the trend of process monitored parameter. The specialists have no unanimous opinion regarding the structures to be used for stability assessment. Western Electric [4,5] four criteria are widely popular; one of them for example is as follows: at least eight successive points located on one side of the central line show the process instability. ISO distinguish eight criteria [6], six criteria are offered for Hotelling's chart [7].

Generalized variance algorithm is based on normal inverted distribution, and as a rule, practical calculations are done on the basis of three sigma rule: in formula (3) we take $u_{1-\alpha / 2}=3$. To find the fault one can use the same specialized structures types as for Schewart's chart. They are: 1) at least one point getting out beyond the control lines, 2) at least two out of three points located on one side of the central line, getting out beyond the twin sigma limits 3) at least four out of five successive points located on one side of the central line, getting out beyond one sigma limit 4) at least eight successive points located on one side of the central line , 5) six decreasing or increasing points in a row (trend), 6) fourteen in turn increasing and decreasing points ( cycles) etc.

The probability of eight points in a row on one side of the central line may be detected as follows. Firstly, we check the criterion fault (6) i.e. a point getting out beyond one of the control lines, probability of this event while using tree sigma rule is equal to $0,0027 / 2=0,00135$. The probability of one point getting to one side of the central line is equal to 0,5 . Then the probability of eight points on one side of the central line provided all the points are located within the control lines is equal to $(0,5-0,00135)^{8}=0,003823$, this is commensurate with the probability of false alert 0,0027 .

Plotting the control charts on PC, the search of specialized structures of any type on the charts is easily computerized, without any difficulties. But, take into account that the increase of criteria number will lead to decrease of observations number among false alerts. Using only structure 1 to detect the unsteady state this number is equal to $1 / \alpha \approx 370$ samples, structures 1 and 4 get 153 samples selecting four structures from the $1^{\text {st }}$ to $4^{\text {th }}$ lead to 92 samples. This value is acceptable, but the use of additional criteria may bring the number of observations among the false alerts to unacceptably small value.

\subsection{Exponentially weighted moving average chart for generalized variance}

To detect the step-wise increase in the dispersion exponentially weighted moving average algorithm for generalized varianceis seldom used; the corresponding values are determined as per formula 
$E_{t}=(1-k) E_{t-1}+k\left|\mathbf{S}_{t}\right|$

where $0 \leq k \leq 1$ is a smoothening parameter, $E_{0}=\left|\Sigma_{0}\right|$. The process is considered steady if the found values are within the control lines

$$
\left.\begin{array}{l}
U C L \\
L C L
\end{array}\right\}=\left|\Sigma_{0}\right| \pm H \sigma_{E t}
$$

where $H$ is a parameter, which determines the location of the control lines; $\sigma_{E t}$ is a mean square deviation of $E_{t}$ values , determined as per formula :

$$
\sigma_{E t}^{2}=\frac{\sigma_{|S|}^{2}}{n} \frac{k}{2-k}\left[1-(1-k)^{2 t}\right] .
$$

Fig. 2 shows the chart of exponentially weighted moving average for generalized variance, plotted with the same data as in Fig. 1 chart. It is seen, that in observations 18-19 there is a fault in the process (intentionally simulated little dispersion), which was not found by the chart of generalized variance.

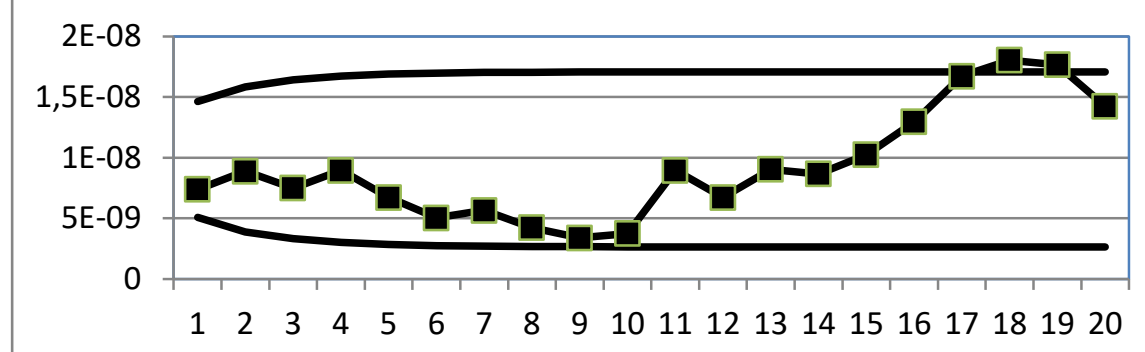

Fig.2. The chart of exponentially weighted moving average for generalized variance.

\subsection{The offered way to assess the object functioning stability}

The conducted investigation made it possible for us to offer the following way of object operation stability assessment: 1. Under the conditions of flawless stable operation the detectors readings are taken and main statistical characteristics are calculated: mean values vector and covariant matrix (characteristics of learning sample).

2. A set of all possible statistical tools is selected for further monitoring. Non correlated data are monitored by the tools based on Schewart's chart. To monitor mean level of correlated parameters, Hotelling's chart is used, to monitor multivariate dispersion, generalized variancechart is used.

3. When necessary the exponentially weighted moving average algorithm based on Hotelling statistics and generalized variance is used.

4. Constant monitoring of object operation is done in order to detect destabilization. Specialized structures are searched on the charts, which prove the possible fault in the process.

\section{Results and discussion}

The computational investigation was done based on the example of hydraulic unit serviceability with the use of vibration dispersion stability criterion [12]. The detectors readings were correlated; simulated dispersion increase was captured by the exponentially weighted moving averages chart (Fig.2)

To assess the stability of object functioning stability as per the criteria of multivariate dispersion one may use the control charts of generalized variance. But these charts do not always detect the faults on time. There were offered methods for the charts sensitivity improvement: the search of non- random structures and the use of algorithmof exponentially weighted moving averages can significantly increase the monitoring efficiency.

\section{Conclusion}

The offered way of object functioning destabilization diagnostics, based on the process statistical control methods, enables timely detection of the operation faults, connected with its parameters dispersion alternation, and prevents an emergency when necessary.

\section{Acknowledgements}

The investigation is done with financial support of RFFI (РФФИ), projects №16-48-732002.

\section{References}

[1] KlyachkinVN, Karpunina IN. The use of statistical control methods to assess units operation stability. Reports of RF Higher School Academy of Science 2016; 3: 65-72. 
[2] Klyachkin VN, Kuvaiskova YuYe, Aleshina AA. Simulating of hydraulic unit vibration on the basis of adaptive dynamic regression. Computerizing. Modern technologies 2014; 1: 30-34.

[3] Kuvaiskova YuYe, Bulyzhev YeM, Klyachkin VN, Bubyr DS. Predict ing the water supply source status in order to ensure water quality. Reference book. Engeneering Journal with attachments 2016; 5: 37-42.

[4] Montgomery DC. Introduction to statistical quality control. New York: John Wiley and Sons, 2009; 754 p.

[5] Ryan TP. Statistical methods for quality improvement. New York: John Wiley and Sons, 2011; 687 p.

[6] Klyachkin VN. Models and methods of statistical control of polyvalent process. M.: Fizmatlit, 2011; 196 p.

[7] Klyachkin VN, Kravtsov YuA. The detection of faults during process multivariate statistical monitoring. Software and systems 2016; 3: 192-197.

[8] Svyatova TI, Klyachkin VN. Multivariate statistical monitoring of dispersion process. Radio technology 2014; 11: 123-126.

[9] Klyachkin VN, Svyatova TI. Methods of statistical monitoring of process as per criteria of multivariate dispersion. Radio industry 2015; 4: 147-153.

[10] García-Díaz Carlos J. The 'effective variance' control chart for monitoring the dispersion process with missing data. Industrial Engineering 2007; 1(1): 4045 . 